\title{
Análisis de los mecanismos de cohesión en cuatro conversaciones en Facebook
}

\author{
David Pérez Retana \\ Departamento de Filosofía, Arte y Letras (FAL) \\ Sede de Occidente, Universidad de Costa Rica
}

\begin{abstract}
Resumen
Este artículo es el resultado de la investigación Mecanismos de cohesión textual en las conversaciones en Facebook, elaborada para optar por el grado maestría en Lingüística de la Universidad de Costa Rica. Específicamente, se analiza cómo interactúan los mecanismos de cohesión textual en cuatro conversaciones de Facebook para determinar de qué manera se logra la cohesión en este tipo de textos, y cómo inciden sobre los cambios temáticos. Para dicho propósito, se comentan en detalle cuatro conversaciones distintas, las cuales son representativas en tanto incluyen casos de los cuatro principales mecanismos de cohesión disponibles en español: conexión, referencia, elipsis y cohesión léxica. El estudio concluye que existen mecanismos textuales especializados en vincular distintas intervenciones en una misma conversación, mientras que otros se orientan a vincular elementos textuales presentes en una misma intervención.
\end{abstract}

Palabras claves: Facebook, cohesión textual, lingüística textual, ciberhabla

\begin{abstract}
This article presents the results of the research Mechanisms of Textual Cohesion in Conversations on Facebook, prepared to qualify for the master degree in Linguistics from the University of Costa Rica. Specifically, it analyzes how the mechanisms of textual cohesion interact in four conversations in Facebook to determine how cohesion is achieved in such texts, and how it impacts on the thematic changes. For this purpose, four different conversations are discussed in detail. Such conversations include the four main mechanisms of cohesion available in Spanish: connection, reference, ellipsis, and lexical cohesion. The study shows the existence of textual mechanisms specialized in linking different interventions into one conversation, while others mechanisms are oriented to linking textual elements in the same intervention.
\end{abstract}

Key words: Facebook, textual cohesion, textual linguistics, Netspeak 


\section{Introducción}

$\mathrm{E}$ $n$ el presente estudio analizaremos la interacción de los mecanismos de cohesión textual en cuatro conversaciones en Facebook, con el fin de determinar de qué manera se logra la cohesión en este tipo de textos y cómo inciden sobre los cambios temáticos. Para dicho objetivo, comentaremos en detalle cuatro conversaciones distintas extraídas de esta red social representativas en tanto incluyen casos de los cuatro principales mecanismos de cohesión en español: conexión, referencia, elipsis y cohesión léxica. Las conversaciones, así como su análisis y resultados, se desprenden de la investigación Mecanismos de cohesión textual en las conversaciones de Facebook, elaborada por el autor para optar por el grado de Magister Linguisticae de la Universidad de Costa Rica.

La importancia del análisis integral de estos mecanismos cohesivos radica en que la estabilidad de un texto, tal y como lo mencionan Beaugrande y Dressler (1997), se mantiene gracias a la continuidad de los elementos que lo integran. Esta continuidad se basa en la suposición de que "cada elemento lingüístico es un instrumento eficaz para acceder a otros elementos lingüísticos" (Beaugrande y Dressler, 1997, p.89). Lo anterior resulta relevante porque, en la mayoría de las conversaciones recopiladas, no se sigue un solo hilo temático, sino que cambia de acuerdo con el hablante; por tanto, nuestro propósito es determinar cómo afecta esta estructura temática, tan particular en las conversaciones de Facebook, la cohesión interna del texto.

\section{Aproximación teórico-metodológica}

A continuación, nos referiremos a los principales conceptos sobre los que se articula esta investigación. Para ello, iniciaremos con una breve descripción de Facebook, red social en la que se basa este estudio.

\section{Caracterización de Facebook}

Facebook es una red social virtual fundada el 4 de febrero de 2004. Cuenta con 1110 millones de usuarios activos y se halla disponible en 110 idiomas (datos correspondientes a marzo de 2013). Sus creadores la definen como "una utilidad social que ayuda a las personas a comunicarse más eficazmente con sus amigos, familiares y compañeros de trabajo" (Facebook.com). Asimismo, su compañía busca desarrollar tecnologías que facilitan el intercambio de información a través del gráfico social y del mapa digital de las conexiones existentes entre las personas en el mundo real (facebook.com/info).

Los principales servicios que esta plataforma ofrece son:

- Lista de amigos (contactos)

- Chat

- Grupos y páginas

- Muro. Se trata de un espacio donde los usuarios pueden escribir y recibir comentarios de sus contactos. Es donde se desarrolla la mayor cantidad de eventos comunicativos.

- Fotos

- Botón de "me gusta"

Para la presente investigación, las conversaciones se extrajeron del "muro" de los usuarios por ser, como se dijo, el espacio donde se da la mayoría de las interacciones. 


\section{Definición de texto según Beau- grande y Dressler}

Beaugrande y Dressler (1997) señalan que, en muchos trabajos realizados desde distintos enfoques teóricos, se suele definir el texto como una unidad superior a la oración, como una distribución discursiva de morfemas o como una secuencia de oraciones correctamente formadas, lo que restringe enormemente su concepto. Debido a lo anterior, estos autores elaboran su propia definición del término, la cual toma en consideración aspectos extralingüísticos.

Un texto es un acontecimiento comunicativo que cumple siete normas de textualidad. Si un texto no satisface alguna de esas normas entonces no puede considerarse que ese texto sea comunicativo. Por consiguiente, los textos que no sean comunicativos no pueden analizarse como si fueran textos genuinos (Beaugrande y Dressler, 1997, p. 35).

Para estos investigadores, un texto es una unidad comunicativa y no solo la suma de palabras, oraciones o párrafos relacionados entre sí. Para ellos, un texto se define por su textualidad y no por su gramaticalidad, de ahí que su propiedad fundamental sea esta condición, la cual debe ser entendida como el conjunto integrado de las siete normas textuales, a saber: la cohesión, la coherencia, la intencionalidad, la aceptabilidad, la informatividad, la situacionalidad y la intertextualidad. La investigación sobre la que se basa este artículo es la cohesión, por tanto, en el siguiente apartado nos referiremos a ella con más detalle.

\section{Cohesión}

Para Beaugrande y Dressler, la cohesión es la que "establece las diferentes posibilidades en que pueden conectarse entre sí dentro de una secuencia los componentes de la superficie textual" (Beaugrande y Dressler, 1997, p.35), entendiéndose como componentes las palabras que conforman el texto. Señalan, asimismo, que dicha conexión en la superficie está en dependencia de las convenciones y formalidades gramaticales de la lengua, donde la sintaxis es la responsable de la cohesión, pues brinda continuidad a los elementos que contribuyen con la estabilidad del texto. Para los autores, cualquier procedimiento lingüístico que opere en función de relacionar los elementos superficiales del texto será parte de la cohesión. Estos mecanismos son los que permiten al lector u oyente interpretar con eficacia el texto, pero aclaran que esta norma no es decisiva por sí misma, sino que debe estar en interacción con las otras normas de textualidad.

\section{Mecanismos de cohesión}

Según Beaugrande y Dressler (1997), en el momento de procesar textos, intervienen distintos mecanismos que permiten la reutilización, la modificación o la compresión de las estructuras y de los patrones usados previamente. Estos mecanismos son: la conexión, la referencia, la elipsis y la cohesión léxica, los cuales contribuyen a la economía del texto y a que su superficie sea estable.

Aunque nuestra principal referencia teórica es la obra de Beaugrande y Dressler (1997), también nos hemos 
apoyado en los aportes de Halliday (2004) quien, en algunos puntos, analiza con mayor profundidad algunos de estos mecanismos en su obra. Seguidamente, nos referiremos a cada uno de estos mecanismos, los cuales serán ilustrados con ejemplos tomados del corpus de la investigación:

- Conexión: para Pons (1998), textualmente, un conector se define por su capacidad de establecer relaciones extraoracionales, por poseer valor deíctico, por ser un instrumento de cohesión textual que jerarquiza las oraciones $u$ otros componentes del texto y por su capacidad para indicar cambios de tópico.

a. "la religión no es importante para mí, pero estoy segura de que Dios existe" (texto 24) (adversación)

b. "mejor nos echamos las birras en Cartucho, porque esa viajadera me frustra" (texto 18) (causal)

c. "Si va a un bautizo, quiere ser el bebé; si va a una boda, quiere ser la novia, $y$ si va a un entierro, quiere ser el muerto" (texto 23) (condicional)

- Referencia: para Beaugrande y Dressler, los mecanismos de referencia "permiten reemplazar elementos independientes portadores de contenido por formas dependientes más breves" (1997, p.91).

a. "(...) son mediocres y no tienen la decencia de aceptarlo [ser mediocres]" (texto 2)

b. “(...) y luego fue encontrado su cuerpo [el de Celia] momificado en 1910" (texto 21)

c. "La única vez que había visto uno antes, fue en San Ramón, pero allá les dicen cuzuco o algo así" (texto 19)
- Elipsis: mecanismo que permite la "repetición incompleta de una estructura y de su contenido, en la que se ha omitido alguna de las expresiones superficiales originales" (Beaugrande y Dressler, 1997, p.91).

a. "Messi sos una diva!!! Una [Ø: razón] más a mi lista de razones para odiar el futbol" (texto 1) (elisión nominal).

b. "Sebas tiene prohibido ver KID vs CAT (...). Después [Ø: Sebas] se vuelve como Ud." (texto 7) (elisión en grupo nominal)

- Cohesión léxica: se trata de la simple reaparición de los elementos superficiales en el texto (Beaugrande y Dressler, 1997). Puede manifestarse en repetición, repetición parcial, paralelismo, paráfrasis y sinonimia.

a. "Que RIDICULAS las viejas que andan pa'rriba y pa'bajo con la empleada y el/la hij@!! Ahora vi una que se bajo del carro con la empleada a recoger al hijo y despúes salió caminando con sus taconcitos de 5 pulgadas y la empleada con el niño en brazos dándole besos. Que Dios me libre de caer en ridiculeces de esas!" (repetición)

b. "Laura: Eso sí, ahorremos para largarnos a tierras exóticas el próximo año.

Ericka: Exotiquémonos -jajajaEsto suena bastante raro para algunos que ya me consideran bastante exótica, pero bueno, you know what I mean :o)" (texto 20) (Repetición parcial)

c. "Silvia: Messi me importa lo mismo q un cacahuete! Puede irse derecho al carajo

Priscilla: Es que yo no se que se cree ese enano" (texto 1) 
d. "Ale: Qué interesante que alguna gente siga creyendo que quienes usan el tratamiento 'usted' son personas viejas o muy muy formales.

Ale: Las personas creen que ud solo es para trato formal $y$ de viejitos" (texto 13) (Paráfrasis)

e. "Joaquín: $\mathrm{Si}$ va a un bautizo, quiere ser el bebé; si va a una boda, quiere ser la novia, y si va a un entierro, quiere ser el muerto Gaby: si va a la ONU, quiere dirigir el consejo de seguridad" (texto 23) (Paralelismo)

\section{Metodología}

Para la conformación del corpus de la tesis, se recopilaron treinta conversaciones de distinta extensión y temática (aprox. 1200 mecanismos cohesivos). Las conversaciones fueron tomadas de los "muros" de las cuentas de los usuarios. Se excluyeron textos provenientes de cuentas de famosos/ figuras públicas, políticos, empresas, instituciones, medios de comunicación, agencias publicitarias, sociedades o asociaciones. Se procuró que los usuarios participantes en las interacciones fueran costarricenses. En cuanto a los temas de conversación, no hubo restricciones; sin embargo, se buscó la variedad temática y se privilegiaron con- versaciones informales. En el caso del presente artículo, nos basamos, con el fin de ilustrar el método utilizado, en cuatro conversaciones.

Con el objeto de facilitar el análisis y comentario de las conversaciones seleccionadas, estas se presentan en una tabla independiente. En sus columnas se indican: el hablante, el texto y los mecanismos cohesivos; en sus filas se presenta cada una de las intervenciones de los hablantes. Los mecanismos cohesivos se señalan en el texto por medio de una numeración consecutiva en superíndice para facilitar su localización en la columna respectiva; asimismo, las intervenciones de cada participante han sido divididas en fragmentos para facilitar las referencias dentro de la conversación; los fragmentos han sido ordenados numéricamente.

A continuación, presentamos la lista de abreviaturas empleadas en el análisis tabular. N: elipsis nominal; V: elipsis verbal; C: elipsis clausal; R: repetición; RP: repetición parcial; S: sinonimia; PL: paralelismo; PF: paráfrasis.

\section{Análisis}

\section{Análisis de la conversación uno}

Participantes: cuatro

Intervenciones: siete

Mecanismos identificados: veintitrés

Tabla 1

Conversación uno

\begin{tabular}{|c|c|c|c|c|c|}
\hline Hablante & & Conexión & Referencia & Elipsis & C. léxica \\
\hline \multirow[t]{4}{*}{ Priscilla } & 1.Messi sos una diva!!! & & & 1. (razón) & \\
\hline & 2.Una $[Ø]^{1}$ más a mi lis- & & & Referencia & \\
\hline & ta de razones & & & catafórica & \\
\hline & 3. para odiar el futbol! & & & vinculada a & \\
\hline
\end{tabular}




\begin{tabular}{|c|c|c|c|c|c|}
\hline \multicolumn{2}{|l|}{ Hablante } & \multirow[t]{2}{*}{ Conexión } & \multirow[t]{2}{*}{ Referencia } & \multirow{3}{*}{$\begin{array}{l}\text { Elipsis } \\
\text { "razones", en } \\
\text { el fragmento } 2 .\end{array}$} & C. léxica \\
\hline & & & & & \\
\hline Benjamin & $\begin{array}{l}\text { 4. jajaja no seas proble- } \\
\text { mática } \\
5 \text {. bien que te } \mathrm{t}^{2} \text { gusta el } \\
\text { futbol }^{3}\end{array}$ & & $\begin{array}{l}2 . \text { te (exofó- } \\
\text { rica) }\end{array}$ & & $\begin{array}{l}\text { 3. futbol. } \\
\text { Apareció por } \\
\text { primera vez en } \\
\text { el fragmento } 3 .\end{array}$ \\
\hline Silvia & $\begin{array}{l}\text { 6. Pri, al fin alguien de } \\
\text { mi }^{4} \text { lado } \\
\text { 7. Messi }{ }^{5} \mathrm{me}^{6} \text { importa lo } \\
\text { mismo q un cacahuete! } \\
\text { 8. Puede irse }{ }^{8} \text { derecho al } \\
\text { carajo }\end{array}$ & & $\begin{array}{l}\text { 4. mi (exofó- } \\
\text { rica) } \\
\text { 6. me (exofó- } \\
\text { rica) } \\
\text { 8. -se (anafó- } \\
\text { rica). Refiere } \\
\text { a Messi (fr. 7) }\end{array}$ & & $\begin{array}{l}\text { 5. Messi. } \\
\text { Apareció } \\
\text { por prime- } \\
\text { ra vez en el } \\
\text { fragmento } 1 .\end{array}$ \\
\hline Laura & 9. Messi ${ }^{9}$ come mierda & & & & $\begin{array}{l}\text { 9. Messi. Ter- } \\
\text { cera mención. }\end{array}$ \\
\hline Priscilla & $\begin{array}{l}\text { 10. Silvia q es esa vara }{ }^{10} \\
\text { de } \text { cacahuate }^{11} \text { ? } \\
\text { 11. Jajaja }\end{array}$ & & $\begin{array}{l}\text { 10. esa vara } \\
\text { (anafórica). } \\
\text { Refiere a ca- } \\
\text { cahuate, en el } \\
\text { fragmento } 7 .\end{array}$ & & $\begin{array}{l}\text { 11. cacahuate. } \\
\text { Mencionado } \\
\text { por primera } \\
\text { vez en el frag- } \\
\text { mento } 7 \text {. }\end{array}$ \\
\hline Priscilla & $\begin{array}{l}\text { 12. jaja no soy proble- } \\
\text { matica }{ }^{12} \text { ! } \\
\text { 13. Es que }{ }^{13} \text { yo }^{14} \text { no se } \\
\text { que se }{ }^{15} \text { cree ese enano }^{16} \text {. } \\
\text { 14. Ahora me } \mathrm{e}^{17} \text { decia } \\
\text { mami que como siempre, } \\
\text { 15. ponen a la Sele en } \\
\text { un pedestal } \\
\text { 16. } \mathrm{y}^{18} \text { los demas atletas } \\
\text { nacionales } \\
\text { 17. que SI ponen en alto } \\
\text { este pais, } \\
\text { 18. tienen que andarla } \\
\text { pulseando } \\
\text { 19. para que } \\
\text { pelota. }\end{array}$ & $\begin{array}{l}\text { 13. Es que } \\
\text { (explicativo). } \\
\text { Amplía la } \\
\text { razón para } \\
\text { odiar a Messi. } \\
\text { 18.y (adición). } \\
\text { Añade nueva } \\
\text { información } \\
\text { al texto. } \\
\text { 19. para que } \\
\text { (finalidad). } \\
\text { Especifica el } \\
\text { fin de “an- } \\
\text { darla pul- } \\
\text { seando". }\end{array}$ & $\begin{array}{l}\text { 14. yo } \\
\text { (exofórica) } \\
\text { 15. se (catafó- } \\
\text { rica). Concuer- } \\
\text { da con "ese } \\
\text { enano", en el } \\
\text { fragmento } 13 . \\
\text { 17. me } \\
\text { (exofórica) } \\
\text { 20. les (anafó- } \\
\text { rica). Refiere } \\
\text { a "los demás } \\
\text { atletas nacio- } \\
\text { nales", men- } \\
\text { cionados en el } \\
\text { fragmento } 16 .\end{array}$ & & $\begin{array}{l}\text { 12. proble- } \\
\text { mática } \\
\text { 16. enano } \\
\text { (S). Si- } \\
\text { nonimia } \\
\text { contextual. } \\
\text { En este caso } \\
\text { reemplaza a } \\
\text { "Messi". }\end{array}$ \\
\hline Silvia & $\begin{array}{l}20 . \text { jaja correccion caca- } \\
\text { huete }^{21} \text { ! } \\
21 . \text { jaja justo } \text { lo }^{22} \text { saque } \\
\text { de } \text { mi }^{23} \text { baul de palabras } \\
\text { rebuscadas }\end{array}$ & & $\begin{array}{l}\text { 22. lo (anafó- } \\
\text { rica). Refiere } \\
\text { al uso de la } \\
\text { "expresión } \\
\text { c achuete". } \\
\text { Responde a la } \\
\text { pregunta plan- } \\
\text { teada en el frag- } \\
\text { mento } 10 \text {. } \\
\text { 23.mi(exofórica) }\end{array}$ & & $\begin{array}{l}\text { 21. cacahue- } \\
\text { te. Mencio- } \\
\text { nado en los } \\
\text { fragmentos } 7 \\
\text { y } 10 \text {. }\end{array}$ \\
\hline
\end{tabular}


En la conversación 1, identificamos un primer eje temático, que inicia con la primera intervención y se extiende hasta la cuarta. El núcleo de este primer eje gira en torno al fútbol y al jugador Messi. Específicamente, en la segunda intervención, el hablante (Benjamin) hace referencia al comentario de la primera hablante (Priscilla). El vínculo se logra por medio de la repetición de la palabra "fútbol" (marca 3, fragmento 5).

En las dos intervenciones posteriores, la referencia al jugador se mantiene activa por medio de marcas de referencia y de cohesión léxica. Por ejemplo, en la tercera intervención, se emplea el pronombre "se" (marca 8, fragmento 8), como referencia anafórica a "Messi", mencionado, por primera vez, en la primera intervención (fragmento 1); además, en el fragmento 7 , se hace referencia al jugador por medio de la repetición léxica (marca 5).

En la quinta intervención, la hablante (Priscilla) hace referencia a la expresión "cacahuate", empleada por otra participante (Silvia) en la tercera intervención. Este giro temático se logra gracias a la referencia anafórica y a la repetición léxica de la expresión (ver marcas 10 y 11, en el fragmento 10).

Seguidamente, la misma hablante de la quinta intervención (Priscilla) introduce un nuevo comentario (sexta intervención); esta vez con referencia a la intervención dos. La conexión entre ambas se logra a partir de la repetición de la palabra "problemática" que había sido utilizada por el segundo participante (Benjamín) en el fragmento 4; asimismo, agrega una explicación a su comentario por medio del conector "es que" (marca 12 , fragmento 13), a la que añade nueva información por medio del conector aditivo " $y$ " (marca 17, fragmento 16).
Finalmente, en la sétima intervención, la participante (Silvia) hace referencia a la quinta intervención. La vinculación entre estas dos intervenciones se logra por medio de la repetición de la palabra "cacahuate".

En la figura 1, retratamos la conexión entre cada una de las intervenciones que conforman la conversación uno.

\section{Figura 1 \\ Relación entre las intervenciones de la conversación uno}

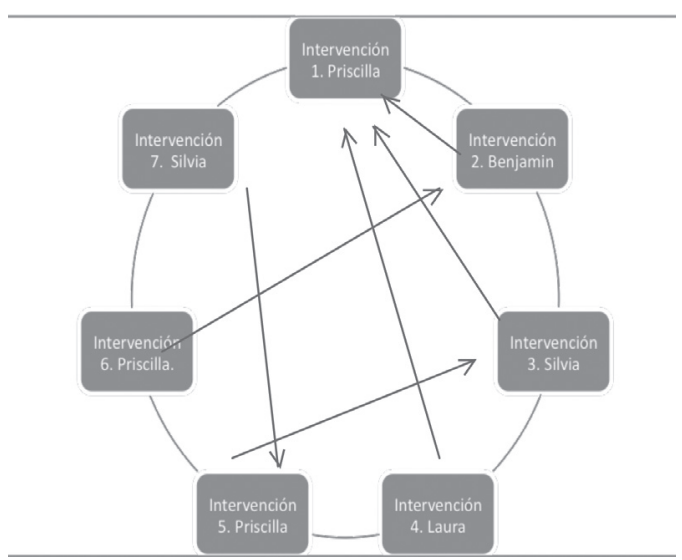

\section{Análisis de la conversación dos}

Participantes: seis

Intervenciones: diez

Mecanismos identificados: treinta

En la conversación dos, identificamos un eje temático principal presente en todas las intervenciones que gira en torno al estado de salud de la primera hablante (Diana). En la segunda intervención, el hablante (Jen) hace referencia a un aspecto específico expuesto en la intervención uno: la forma de respirar. Este enlace se logra gracias a la repetición de las formas "la mayoría de la gente" (marca 5, fragmento 6) y 
"respirar" (marca 6, fragmento 7). Seguidamente, en la siguiente intervención, la hablante (Diana) hace referencia directa a la segunda intervención; el vínculo textual entre ambas se logra mediante la forma anafórica "eso" (marca 11, fragmento 8), que remite a la manera de respirar (fragmento 7).

Las intervenciones cuatro, cinco y seis presentan reacciones ante el contenido de la intervención uno. En el caso de la intervención seis, el hablante (Waldo) apela directamente a la hablante de la intervención uno mediante una pregunta que se responde en la sétima intervención. La vinculación entre ambas intervenciones se logra por medio de procesos de inferencia, pues al ser la intervención seis una pregunta, suponemos que la intervención siete es su respuesta, ya que no hay marcas cohesivas o referencias exofóricas que establezcan una conexión textual entre estas intervenciones. Para lo anterior, nos apoyamos en el concepto de par adyacente, que se define como

dos turnos conversacionales consecutivos que se caracterizan porque la presencia de la primera parte (el primer turno) hace que se espere a continuación una segunda parte determinada: por ejemplo, a un turno del tipo [Hola, zqué tal?] sigue uno como [Bien, ¿̇y tú?]. El par adyacente, pues, es la pareja mínima de la conversación (Tusón, 1997, p.58).

Este mismo principio podría aplicarse a la intervención nueve, mientras que la octava y la décima intervenciones constituyen comentarios de los hablantes ante la intervención uno.

Tabla 2

Conversación dos

\begin{tabular}{|c|c|c|c|c|c|}
\hline Hablante & & Conexión & Referencia & Elipsis & C. léxica \\
\hline Diana & $\begin{array}{l}\text { 1.He de decir, que me } \\
\text { siento muy feliz } \\
\text { 2. de poder respirar como } \\
\text { la mayoria de la gente } l^{1} \\
\text { hace todos los dias } \\
\text { 3. } y^{2} \text { ni siquiera se da cuenta } \\
\text { que }[\varnothing]^{4} \text { es una bendicion. } \\
\text { 4. Tengo fe en que } \mathrm{me}^{5} \text { cu- } \\
\text { rare al } 100 \%:)\end{array}$ & $\begin{array}{l}2 . \quad \text { y (adi- } \\
\text { ción). Añade } \\
\text { nueva infor- } \\
\text { mación al } \\
\text { comentario. }\end{array}$ & $\begin{array}{l}\text { 1. lo (anafó- } \\
\text { rica) Refiere } \\
\text { a la acción } \\
\text { de respirar. } \\
\text { 5. me } \\
\text { (exofórica) }\end{array}$ & $\begin{array}{l}\text { 4. (respirar } \\
\text { como la ma- } \\
\text { yoría de la } \\
\text { gente) }(\mathrm{V})\end{array}$ & \\
\hline Jen & $\begin{array}{l}\text { 5. La mayoría de la } \text { gente }^{6} \\
\text { 6. aquí }{ }^{6} \text { en } \text { mi }^{8} \text { casa } \\
\text { 7. respira }{ }^{9} \text { con la jeta } \\
\text { abierta... jajaja }\end{array}$ & & $\begin{array}{l}\text { 7. aquí } \\
\text { (exofórica) } \\
\text { 8. mi (exo- } \\
\text { fórica) }\end{array}$ & & $\begin{array}{l}\text { 6. La ma- } \\
\text { yoría de la } \\
\text { gente (R). } \\
\text { S e g u n d a } \\
\text { m e n c ión. } \\
\text { Aparece por } \\
\text { primera vez } \\
\text { en el frag- } \\
\text { mento } 1 . \\
9 . \quad \text { respira } \\
\text { (RP). Aparece }\end{array}$ \\
\hline
\end{tabular}




\begin{tabular}{|c|c|c|c|c|c|}
\hline Hablant & & Conexión & Referencia & Elipsis & C. léxica \\
\hline Diana & $\begin{array}{l}\text { 8. Bueno }{ }^{10} \text { mae, eso }{ }^{11} \text { es } \\
\text { por irritaciones en las vias } \\
\text { respiratorias } \\
9 . \mathrm{y}^{12}[\varnothing]^{13} \text { no es normal! } \\
\text { 10. Te }{ }^{14} \text { juro que el trata- } \\
\text { miento que me }{ }^{15} \text { mando el } \\
\text { Dr. SI es caro } \\
\text { 11. pero }{ }^{16} \text { no he estado libre } \\
\text { de inhalador en muchos } \\
\text { años dos dias seguidos! } \\
\text { 12. Asi que }{ }^{17}, \text { bronquitis, } \\
\text { chau, asma, chau }{ }^{18}, \text { rinitis } \\
\text { cuando el asma }{ }^{19} \text { chau }^{20} \text {, } \\
\text { chau }^{21} !>.<\end{array}$ & $\begin{array}{l}\text { 10. bueno (co- } \\
\text { mentador). } \\
\text { Pr e s e n t a } \\
\text { un nuevo } \\
\text { comentario } \\
\text { asociado a la } \\
\text { primera in- } \\
\text { tervención. } \\
\text { 12. y (adición). } \\
\text { Agrega in- } \\
\text { for ma ción } \\
\text { nueva, aun- } \\
\text { que tam- } \\
\text { bién podría } \\
\text { atribuírsele } \\
\text { una función } \\
\text { adversativa. } \\
\text { l6. pero (ad- } \\
\text { versación). } \\
\text { Establece una } \\
\text { relación con- } \\
\text { traargumen- } \\
\text { tativa con } \\
\text { respecto al } \\
\text { fragmento } 10 . \\
\text { l7. Así que } \\
\text { (consecuencia). } \\
\text { Presenta un } \\
\text { re s u l a do } \\
\text { asociado a } \\
\text { los fragmen- } \\
\text { tos } 10 \text { y } 11 .\end{array}$ & $\begin{array}{l}\text { 11. eso (ana- } \\
\text { fóri-ca). Re- } \\
\text { fiere a "res- } \\
\text { pirar con la } \\
\text { jeta abier- } \\
\text { ta" (frag- } \\
\text { mento 7). } \\
\text { 14. te } \\
\text { (exofórica) } \\
\text { 15. me } \\
\text { (exofórica) }\end{array}$ & $\begin{array}{l}\text { 13. (respirar } \\
\text { con la jeta } \\
\text { a bi e r ta). } \\
(\mathrm{V}) \text {. Asocia- } \\
\text { do a la in- } \\
\text { tervención } \\
\text { a n t e rior } \\
\text { (fragmento7). }\end{array}$ & $\begin{array}{l}\text { por prime- } \\
\text { ra vez en el } \\
\text { fragmento } 2, \\
\text { en su forma } \\
\text { de infinitivo. } \\
\text { 18. chau (R). } \\
\text { Se menciona } \\
\text { en el frag- } \\
\text { mento } 12 . \\
\text { 19. asma (R). } \\
\text { Se menciona } \\
\text { en el frag- } \\
\text { mento } 12 . \\
20 . \text { chau (R). } \\
\text { Se menciona } \\
\text { en el frag- } \\
\text { mento } 12 . \\
21 . \quad \text { chau } \\
\text { (R). Se men- } \\
\text { ciona en el } \\
\text { frag a ento } \\
\text { 12. } \\
\text { *Los casos } \\
\text { 17, } 19 \text { y } 20 \\
\text { p o d r í a n } \\
\text { considerar- } \\
\text { se, también, } \\
\text { un caso de } \\
\text { paralelismo. }\end{array}$ \\
\hline Adriana & $\begin{array}{l}\text { 13. Exito!!!! :) } \\
\text { 14. Que bueno saber que }\end{array}$ & & $\begin{array}{l}22 . \text { te } \\
\text { (exofórica) }\end{array}$ & & \\
\hline
\end{tabular}

Diana 15. Ay adri! Gracias!

Waldo 16. que tienes Diana?

Diana 17. $\mathrm{Me}^{23}$ cayeron las 7 plagas! 18. Pero ${ }^{24}$, la $[\varnothing]^{25}$ que $\mathrm{lo}^{26}$

24. pero (ad- 23. me 25.(plaga)(N). 27. bronquicoronó fue una bronquitis ${ }^{27}$ versación). (exofórica) Se menciona tis $(\mathrm{R})$. aguda!!! Y asmática ${ }^{28}$ :) Establece 26. lo en el frag- $\mathrm{S}$ e g u n d a una relación (exofórica) mento 17 . mención; 


\begin{tabular}{|c|c|c|c|c|c|}
\hline Hablante & & Conexión & Referencia & Elipsis & C. léxica \\
\hline & & $\begin{array}{l}\text { contraargu- } \\
\text { mentativa } \\
\text { con respecto } \\
\text { al fragmen- } \\
\text { to } 17 \text {. }\end{array}$ & & & $\begin{array}{l}\text { la primera } \\
\text { aparece en el } \\
\text { fragmento } 12 \text {. } \\
28 \text {. asmática } \\
(\mathrm{RP})\end{array}$ \\
\hline Karina & 19. : ( & & & & \\
\hline Waldo & $\begin{array}{l}\text { 20. q sigas mejor } y^{29} \text { cui- } \\
\text { date }^{30}\end{array}$ & $\begin{array}{l}29 . \text { y (adi- } \\
\text { ción). Añade } \\
\text { información. }\end{array}$ & $\begin{array}{l}\text { 30.te } \\
\text { (exofórica) }\end{array}$ & & \\
\hline Elena & $\begin{array}{l}\text { 21. que te }{ }^{31} \text { mejores pron- } \\
\text { to Dianita!! :) }\end{array}$ & & $\begin{array}{l}\text { 31. te (exo- } \\
\text { fórica) }\end{array}$ & & \\
\hline
\end{tabular}

En la figura 2, presentamos la relación entre cada una de las intervenciones que componen la conversación dos.

Figura 2

Relación entre las intervenciones de la conversación dos

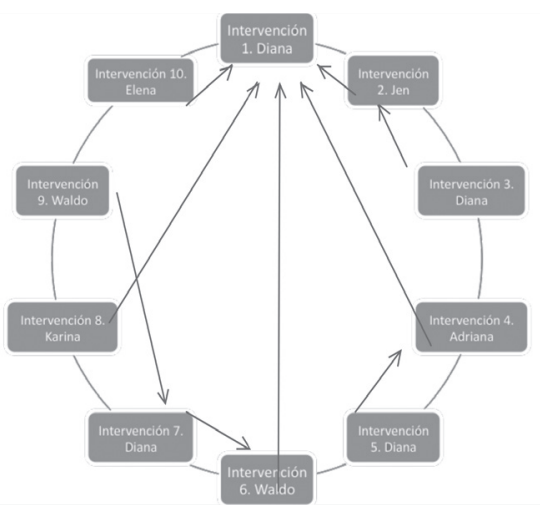

\section{Análisis de la conversación tres}

Participantes: cuatro

Intervenciones: cuatro

Mecanismos identificados: quince

En el caso de la tercera conversación, identificamos dos ejes temáticos presentados desde la primera intervención y relacionados entre sí. El primero obedece a la inauguración del Estadio Nacional; el segundo, a la presencia de Óscar Arias (Presidente de la República) y Laura (Presidenta de la República electa: Laura Chinchilla) en el evento. Las intervenciones dos y tres se vinculan con el primero de los temas, mientras que la intervención cuatro lo hace con el segundo.

En el caso de la segunda intervención, la hablante (Priscilla) inicia su intervención con el conector consecutivo "entonces" (marca 4, fragmento 5), por medio del cual liga su comentario con los fragmentos 3 y 4 de la primera intervención. Asimismo, la elipsis verbal de "ir al cielo", en el fragmento 5 (marca 6 ), es una referencia anafórica que remite al fragmento 4 de la intervención previa. Asimismo, el vínculo de esta intervención con el segundo de los temas señalados (la presencia de Óscar Arias y Laura) se da por medio del uso del pronombre "ellos" (fragmento 6, marca 8).

Por su parte, la tercera intervención establece un vínculo con la primera, con respecto a la presencia de "Óscar Arias" y "Laura", por medio del empleo del pronombre "les" (fragmento 10 , marca 12), con el cual se refiere anafóricamente a ambos personajes políticos en Costa Rica. 
Finalmente, la cuarta intervención se vincula con la primera al hacer referencia a la inauguración del Estadio Nacional. Esta conexión se logra gra- cias a la cohesión léxica, específicamente mediante la repetición de los vocablos "estadio" (fragmento 11, marca 13) e "inauguración" (fragmento 12, marca 14).

Tabla 3

Conversación tres

\begin{tabular}{|c|c|c|c|c|c|}
\hline Hablante & & Conexión & Referencia & Elipsis & C. léxica \\
\hline Ana & $\begin{array}{l}\text { 1. Como demonios } \\
\text { la bestia de OSCAR } \\
\text { ARIAS va a dar un } \\
\text { discurso en la inau- } \\
\text { guración del estadio } \\
\text { nacional? } \\
\text { 2. No es suficiente } \\
\text { el martirio con solo } \\
\text { LAURA?... } \\
\text { 3.tengo malos senti- } \\
\text { mientos hacia esos } \\
\text { dos ( y unos cuan- } \\
\text { tos mas) } \\
4 \text {. por eso }{ }^{2} \text { no me }{ }^{3} \text { voy } \\
\text { a ir al cielo jajajaja }\end{array}$ & $\begin{array}{l}\text { 2. por eso (con- } \\
\text { secutivo). Esta- } \\
\text { blece de causa/ } \\
\text { consecuencia } \\
\text { entre el frag- } \\
\text { mento } 3 \text { y el } \\
\text { fragmento } 4 .\end{array}$ & $\begin{array}{l}\text { 1. esos dos } \\
\text { (anafórica). } \\
\text { Refiere a Ós- } \\
\text { car Arias } \\
\text { (fragmento 1) } \\
\text { y Laura (frag- } \\
\text { mento 2). } \\
\text { 3. me (exofó- } \\
\text { rica) }\end{array}$ & & \\
\hline Priscilla & $\begin{array}{l}\text { 5. entonces }{ }^{4} \mathrm{yo}^{5} \text { tm- } \\
\text { poco }[\emptyset]^{6} \text { ! } \\
\text { 6. Pero } \text { ellos }^{8} \text {, mas } \\
\text { el hermano de ese } \\
\text { orejon playo }{ }^{9} \text { (y } \\
\left.\text { unos cuantos mas }{ }^{10}\right) \\
\text { 7. tiene entrada } \\
\text { VIP PLATINIUM } \\
\text { para el infierno } \\
\text { 8. y asientos a la } \\
\text { pura par de SATA- } \\
\text { NAS! }\end{array}$ & $\begin{array}{l}\text { 4. entonces (con- } \\
\text { secutivo). Vincu- } \\
\text { la la intervención } \\
\text { en la que aparece } \\
\text { con la interven- } \\
\text { ción previa, al } \\
\text { establecer una } \\
\text { relación de con- } \\
\text { secuencia, especí- } \\
\text { ficamente con los } \\
\text { fragmentos } 3 \text { y } 4 \text {. } \\
7 . \quad \text { Pero (ad- } \\
\text { v e r s a c i ó n ). } \\
\text { Establece una } \\
\text { relación contra- } \\
\text { argumen-tativa } \\
\text { con los frag- } \\
\text { mentos } 3,4 \text { y } 5 .\end{array}$ & $\begin{array}{l}\text { 5. yo (exofóri- } \\
\text { ca) } \\
\text { 8. ellos (ana- } \\
\text { fórica). Re- } \\
\text { fiere a Óscar } \\
\text { (fragmento 1) } \\
\text { y Laura (frag- } \\
\text { mento 2). }\end{array}$ & $\begin{array}{l}6 . \quad(\text { voy a } \\
\text { ir al cielo) } \\
(V) \text { Men- } \\
\text { cionado en } \\
\text { el fragmen- } \\
\text { to } 4 .\end{array}$ & $\begin{array}{l}9 . \quad \text { orejón } \\
\text { playo (S). Si- } \\
\text { nonimia con- } \\
\text { textual. Re- } \\
\text { fiere a Óscar } \\
\text { Arias, men- } \\
\text { cionado en el } \\
\text { fragmento } 1 \\
\text { por primera } \\
\text { vez. } \\
\text { 10. y unos } \\
\text { cuantos más } \\
\text { (R). Segun- } \\
\text { da mención. } \\
\text { Mencionado } \\
\text { por prime- } \\
\text { ra vez en el } \\
\text { fragmento } 3 .\end{array}$ \\
\hline Melissa & $\begin{array}{l}\text { 9. ahora si compro } \\
\text { entraas } \\
\text { 10. solo para }{ }^{11} \text { tirar- } \\
\text { les }^{12} \text { tomates y hue- } \\
\text { vos podrideeeos!!! }\end{array}$ & $\begin{array}{l}\text { 11. para (fina- } \\
\text { lidad). Indica } \\
\text { el propósito de } \\
\text { "comprar entra- } \\
\text { das" (frag. 9). }\end{array}$ & $\begin{array}{l}\text { 12. -les (ana- } \\
\text { fórica). Re- } \\
\text { fiere a Óscar } \\
\text { Arias y Laura, } \\
\text { mencionados }\end{array}$ & & \\
\hline
\end{tabular}




\begin{tabular}{|c|c|c|c|c|c|}
\hline Hablante & & Conexión & Referencia & Elipsis & C. léxica \\
\hline Randall & $\begin{array}{l}\text { 11. tanta bulla con } \\
\text { ese estadio } \\
\text { 12. el pichazo de } \\
\text { plata en la inaugu- } \\
\text { racion }^{14} \\
\text { 13. } \text { y }^{15} \text { el san juan } \\
\text { de dios cayendose a } \\
\text { pedazos }\end{array}$ & $\begin{array}{l}\text { 15. y (adición). } \\
\text { Añade nueva } \\
\text { información a } \\
\text { los fragmentos } \\
11 \text { y } 12 \text {. }\end{array}$ & $\begin{array}{l}\text { en la interven- } \\
\text { ción uno (frag- } \\
\text { mentos } 1 \text { y } 2 \text { ). }\end{array}$ & & $\begin{array}{l}13 . \quad \text { estadio } \\
(\mathrm{R}) \text {. Segun- } \\
\text { da mención. } \\
\text { Aparece por } \\
\text { primera vez } \\
\text { en la primera } \\
\text { intervención } \\
\text { (fragmento } 1) . \\
\text { 14. inaugu- } \\
\text { ración (R). } \\
\text { Segunda men- } \\
\text { ción. Aparece } \\
\text { por primera } \\
\text { vez en la pri- } \\
\text { mera interven- } \\
\text { ción (frag. } 1 \text { ). }\end{array}$ \\
\hline
\end{tabular}

Las relaciones entre las intervenciones de la conversación tres podemos representarlas por medio de la figura 3 .

Figura 3

Relación entre las intervenciones de la conversación tres

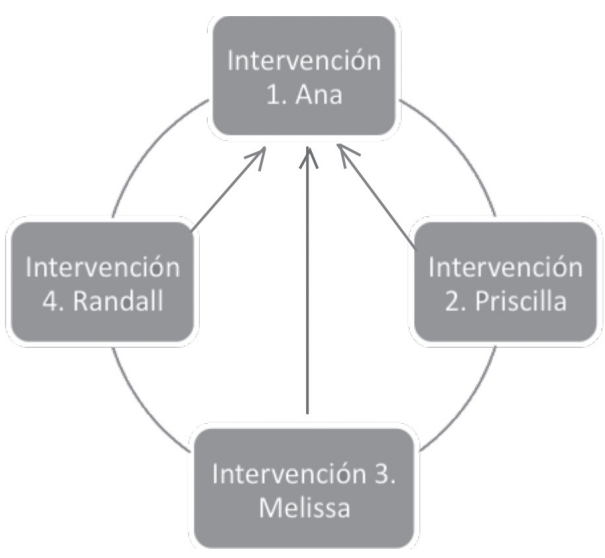

\section{Análisis de la conversación cuatro}

Participantes: tres

Intervenciones: cinco

Mecanismos identificados: veintinueve

La conversación cuatro inicia con el comentario de la hablante (Ericka) sobre un armadillo que observó mientras caminaba. En la segunda intervención, la hablante (Rita) añade un nuevo comentario al respecto, que se vincula con la primera intervención por medio de "y" (marca 1, fragmento 3), que funciona, en este caso, como un comentador. Seguidamente, en la tercera intervención, la hablante (Laura) comenta sobre el mismo tema. $\mathrm{Su}$ intervención es introducida por "ni" (marca 2, fragmento 4). Además, logra establecer la conexión entre su intervención y la primera por medio de la repetición parcial (marca 5 , fragmento 4) de la forma "armadillito bebé", que 
aparece por primera vez en el fragmento 2 de la primera intervención.

En la tercera intervención, la hablante (Ericka) hace referencia, en primer lugar, a la segunda intervención. Dicha referencia se logra gracias a la repetición léxica del vocablo "fotos" (marca 7, fragmento 7), que había aparecido originalmente en el fragmento 3 de la conversación. Luego, la hablante amplía su comentario en la misma intervención y habla sobre la primera vez que había visto un armadillo. Finalmente, se refiere a la tercera intervención; el nexo se da por medio de la repetición de la expresión "la casa de sus papás" (marca 17, fragmento 12), cuya primera aparición se registra en el fragmento 5 .

En la última de las intervenciones, la hablante (Laura) hace referencia a la cuarta intervención. La conexión entre ambas se logra por medio de la repetición léxica (marca 25, fragmento 15) de la forma "parece", que había sido utilizada en el fragmento 14, en la intervención previa. Asimismo, su comentario constituye una corrección de lo afirmado en la intervención cuatro; tal corrección se manifiesta por medio del uso del reformulador rectificativo "más bien" (marca 26, fragmento 15).

Tabla 4

Conversación cuatro

\begin{tabular}{|c|c|c|c|c|c|}
\hline Hablante & & Conexión & Referencia & Elipsis & C. léxica \\
\hline Ericka & $\begin{array}{l}\text { 1. ¡La ternura! } \\
\text { 2. Un armadillito } \\
\text { bebé andaba cami- } \\
\text { nando cerca de mi } \\
\text { casa esta mañana }\end{array}$ & & & & \\
\hline Rita & 3. $Y^{1}$ las fotos? & $\begin{array}{l}\text { 1. Y (comenta- } \\
\text { dor). Pese a que, } \\
\text { en la mayoría de } \\
\text { los casos, "y" aña- } \\
\text { de nueva informa- } \\
\text { ción al texto, en } \\
\text { este caso, su em- } \\
\text { pleo introduce un } \\
\text { nuevo comentario } \\
\text { en la conversación. }\end{array}$ & & & \\
\hline Laura & $\begin{array}{l}\text { 4. } N i^{2} t e^{3} \text { cuento } \\
\text { lo que le pasó a } \\
\text { un armadillo bebé } \\
\text { 5. que entró al pa- } \\
\text { tio de la casa de } \\
\text { mis papás. } \\
\text { 6. :( Fue horrible } \\
\text { fue horrible }{ }^{6} . .\end{array}$ & $\begin{array}{l}2 . \quad \mathrm{Ni} \text { (adición). } \\
\text { Añade nueva in- } \\
\text { formación al texto } \\
\text { presentado en la } \\
\text { primera interven- } \\
\text { ción. }\end{array}$ & $\begin{array}{l}\text { 3. te (exofórica) } \\
4 . \quad \text { le (catafó- } \\
\text { rica). Refiere } \\
\text { a "armadillo } \\
\text { bebé" (frag- } \\
\text { mento 4). }\end{array}$ & & $\begin{array}{l}5 . \quad \text { armadillo } \\
\text { bebé (RP). Se } \\
\text { vincula con el } \\
\text { fragmento } 2 \text { (in- } \\
\text { tervención } 1) \text {. } \\
6 \text {. fue horrible } \\
(\mathrm{R}) \text {. Segunda } \\
\text { mención; apa- } \\
\text { rece en el mis- } \\
\text { mo fragmento. }\end{array}$ \\
\hline
\end{tabular}




\begin{tabular}{|c|c|c|c|c|c|}
\hline Hablante & & Conexión & Referencia & Elipsis & C. léxica \\
\hline Ericka & 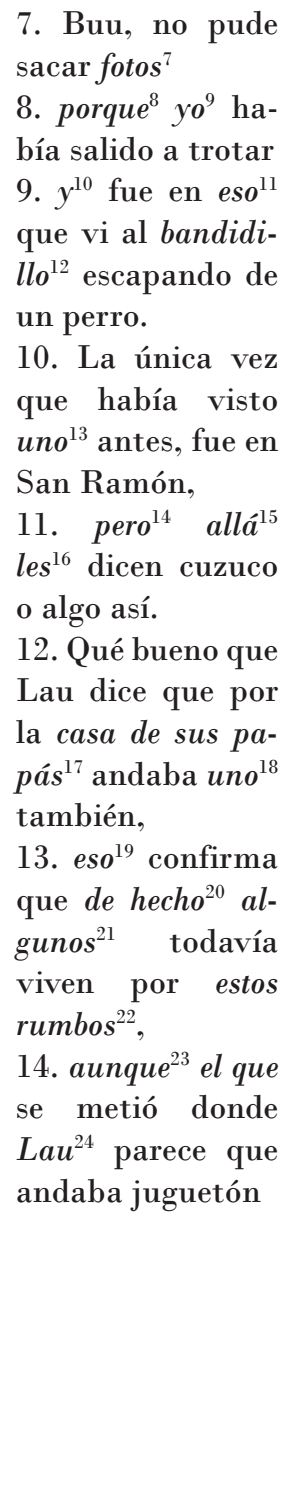 & $\begin{array}{l}\text { 8. porque (causati- } \\
\text { vo). Establece una } \\
\text { relación causal con } \\
\text { el fragmento } 7 . \\
10 . \text { y (adición). } \\
\text { Agrega nueva in- } \\
\text { formación a la in- } \\
\text { tervención. } \\
\text { 14. pero (adversa- } \\
\text { ción). Establece } \\
\text { una relación con- } \\
\text { traargumentativa } \\
\text { con el fragmento } 10 . \\
20 . \text { de hecho (ope- } \\
\text { rador de refuerzo } \\
\text { argumentativo). } \\
\text { Refuerza la afir- } \\
\text { mación presenta- } \\
\text { da en el fragmento } \\
13 . \\
23 . \text { aunque (con- } \\
\text { cesivo). Establece } \\
\text { una relación con- } \\
\text { traargumentativa } \\
\text { con los fragmentos } \\
12 \text { y } 13 \text {. }\end{array}$ & $\begin{array}{l}\text { 9. yo (exofóri- } \\
\text { ca) } \\
\text { 11. eso (anafó- } \\
\text { rica). Funciona } \\
\text { como una mar- } \\
\text { ca temporal. } \\
\text { Alude al mo- } \\
\text { mento en que } \\
\text { salió a trotar. } \\
\text { 13. uno (anafó- } \\
\text { rica). Marca no } \\
\text { correferencial. } \\
\text { 15. allá (ana- } \\
\text { fórica). Refiere } \\
\text { a San Ramón, } \\
\text { en el fragmento } \\
\text { 10. } \\
\text { 16. les (anafó- } \\
\text { rica). Refiere a } \\
\text { "armadillos". } \\
\text { 18. uno (anafó- } \\
\text { rica). Un arma- } \\
\text { dillo. Marca no } \\
\text { correferencial. } \\
\text { 19. eso (anafó- } \\
\text { rica). Refiere a } \\
\text { la información } \\
\text { presentada en } \\
\text { el fragmento } \\
\text { 12. } \\
21 . \\
\text { (a n a fórica). } \\
\text { Refiere a "ar- } \\
\text { madillos". } \\
22 . \text { estos rum- } \\
\text { bos (exofórica). }\end{array}$ & & $\begin{array}{l}\text { 7. fotos (R). } \\
\mathrm{S} \text { e g u n d a } \\
\text { m e n c i ó } \mathrm{n} \text {. } \\
\text { Aparece por } \\
\text { primera vez } \\
\text { en la segunda } \\
\text { intervención } \\
\text { (fragmento 3). } \\
\text { l2. bandidillo } \\
\text { (S). Sinonimia } \\
\text { contex tual } \\
\text { equivalente a } \\
\text { "un armadi- } \\
\text { llito", men- } \\
\text { cionado en la } \\
\text { primera inter- } \\
\text { vención (frag- } \\
\text { mento } 2 \text { ). } \\
\text { l7. la casa } \\
\text { de sus papás } \\
\text { (R). Segun- } \\
\text { da mención. } \\
\text { Aparece por } \\
\text { primera vez } \\
\text { en la tercera } \\
\text { intervención } \\
\text { (fragmento } 5 \text { ). } \\
24 \text {. Lau (R). } \\
\mathrm{S} \text { e g u n d a } \\
\text { m e n c i ó n . } \\
\text { Aparece por } \\
\text { primera vez } \\
\text { en el frag- } \\
\text { mento } 12 \text { de } \\
\text { la cuarta in- } \\
\text { tervención. }\end{array}$ \\
\hline Laura & $\begin{array}{l}\text { 15. } \text { Parece }^{25} \text { más } \\
\text { bien }^{26} \text { que }[\varnothing]^{27} \\
\text { andaba suicida }\end{array}$ & $\begin{array}{l}26 . \text { más bien (re- } \\
\text { formulador rectifi- } \\
\text { cativo) }\end{array}$ & & $\begin{array}{l}\text { 28. (el ar- } \\
\text { madillo) } \\
\text { (N). Se } \\
\text { relacio- } \\
\text { na con el } \\
\text { fragmento } \\
\text { 14. }\end{array}$ & $\begin{array}{l}25 . \quad \text { parece } \\
(\mathrm{R}) \text { Segun- } \\
\text { da mención. } \\
\text { Aparece por } \\
\text { primera vez } \\
\text { en el fragmen- } \\
\text { to } 14 \text {, cuarta } \\
\text { intervención. }\end{array}$ \\
\hline
\end{tabular}

Las relaciones entre las intervenciones de la conversación cuatro se exponen en la figura 4. 
Figura 4

Relación entre las intervenciones de la conversación cuatro

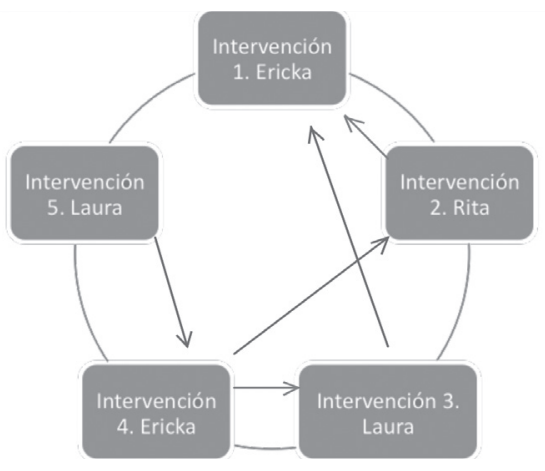

\section{Conclusiones}

Cabe destacar que dadas las limitantes de espacio, en este artículo solo se expuso el análisis detallado de cuatro conversaciones, con el fin de ilustrar la metodología utilizada en el estudio; sin embargo, las conclusiones ofrecidas en esta sección se desprenden del corpus total recopilado para la investigación que origina este artículo (Pérez, 2014), con la intención de proporcionar mayor validez a los resultados.

En primer lugar, podemos afirmar que la cohesión léxica es la principal responsable de establecer relaciones entre las distintas intervenciones que conforman una conversación. Según nuestros datos, la vinculación entre dos intervenciones se logra en $51 \%$ de las veces por medio de la cohesión léxica, es decir, en más de la mitad de los casos.

Para esta misma función, la referencia también ocupa un lugar importante, dado que es el segundo mecanismo más utilizado, con una frecuencia de aparición del $28 \%$. Por su parte, los dos mecanismos restantes, la elipsis y la conexión, parecen desempeñar un papel menos relevante a la hora de vincular intervenciones distintas, lo cual se ve reflejado en su frecuencia de aparición, $12 \%$ para la elipsis y $9 \%$ para la conexión; sin embargo, como detallaremos más adelante, ambas tienen un mayor desempeño en el momento de establecer relaciones dentro de una misma intervención.

Al profundizar sobre el papel que desempeña la cohesión léxica en la vinculación de las intervenciones, notamos que su uso recae notablemente sobre la repetición. De los distintos mecanismos léxicos analizados, la repetición abarca el $73 \%$ de los casos identificados, porcentaje que llegaría a $87 \%$ si se toma en consideración la repetición parcial.

Dado lo anterior, podríamos plantear -aunque se requiere de un estudio más exhaustivo para su afirmación-, que cuanto más alejados estén discursivamente los elementos lingüísticos vinculados, más necesario será el empleo de formas que retomen el contenido semántico con mayor precisión. En tal caso, la reutilización de formas por medio de la repetición sería el mecanismo que mejor cumple esta función, al tratarse de la forma más directa de cohesión.

La repetición de palabras o expresiones idénticas puede poseer distintas motivaciones en cuanto a su uso. Para van Dijk (en Beaugrande y Dressler, 1997), su función es apoyar la coherencia discursiva; para Beaugrande y Dressler (1997), su uso en el habla espontánea es el resultado del breve tiempo de que disponen los hablantes para "planificar la efímera superficie textual". En nuestro caso, como ya se sugirió, consideramos que su función es indicar de forma precisa al lector con cuál o con cuáles intervenciones se relaciona su participación dentro del discurso. 
Con respecto a los mecanismos de cohesión que operan dentro de cada una de las intervenciones, la función recae principalmente sobre la conexión. De acuerdo con los datos obtenidos, la conexión representa el $48 \%$ de los mecanismos cohesivos empleados por los hablantes. El uso principal está asociado a la adición, pero también son frecuentes otros usos, como la contrargumentación y la reformulación.

Finalmente, queremos señalar que en el momento de establecer vínculos entre distintas intervenciones o relacionar información dentro de una misma de estas, la referencia es el segundo mecanismo más utilizado. Su uso representa un 28\% para el primer caso y $24 \%$ para el segundo. Por su parte, indistintamente de su función, la elipsis es el mecanismo cohesivo menos empleado en las conversaciones de Facebook, aunque su uso es levemente superior cuando se trata de establecer vínculos entre intervenciones distintas.

\section{Bibliografía}

Beaugrande, R. y Dressler, W. (1997). Introducción a la lingüística del texto. Barcelona: Editorial Ariel.

Betancourt, A. (2009). El ciberlenguaje, una variedad para tener en cuenta. Interacción, 10 (3), 8-11.

Cassany, D. (2000). De lo análogo a lo digital. El futuro de la enseñanza de la composición. Lectura y vida. Revista Latinoamericana de lectura, 21(2), 2-10.

(2006). Tras las líneas. Barcelona: Editorial Anagrama.

Crystal, D. (2002). El lenguaje e Internet. Madrid: Cambridge University Press.
Erickson, T. (1997). Social Interaction on the Net: Virtual Community as Participatory Genre. En: Proceedings of the Thirtieth Hawaii International Conference on System Science. January, Vol. VI, pp. 1321, Mauai Hawaii. Recuperado el 4 de mayo de 2010 de http://www. pliant.org/personal/Tom_Erickson

Flores, T. (2006). A produção de sentidos em hipertextos: os hiperlinks como anáforas. Tesis de maestría en Lingüística. Universidade Federal do Rio Grande do Sul.

Giannoccaro, P. (2007). Redes Sociais na Internet: as comunidades e blogs como oportunidades para publicidade e o marketing. Tesis de maestría en Administración. Pontifícia Universidade Católica de São Paulo.

Halliday, M. (2004). An Introduction to Functional Grammar. London: Hodder Arnold.

Halliday, M. y Hasan, R . (1976). Cohesion in English. London y Nueva York: Longman.

Roche, X. (s.f.). HTTrack Website Copier. En www.httrack.com

Laborda, X. (2004). Foros virtuales, ética lingüística y aspectos legales. RED. Revista de Educación a Distancia. III (12). Recuperado de: http://www.um.es/ead/red/12/

(2005). Tecnologías, redes y comunicación interpersonal. Efectos en las formas de la comunicación digital. Anales de documentación. No 8. 101-116. ISSN (versión en línea): 1697-7904. http://revistas. um.es/analesdoc/article/view/1511

Marcuschi, L. (1999). Linearização, cognição e referência: o desafio do hipertexto. En: Línguas e Instrumentos Lingüísticos. 3. Campinas: Editora Pontes. Recuperado el 6 de 
marzo de 2010 de: http://www.pucsp. br/ fontes/ln2sem2006/17Marcus. pdf

(2001). O hipertexto como um novo espaço de escrita em sala de aula. Linguagem \& Ensino, 4 (1), 79-111.

(2002). Gêneros textuais emergentes no contexto da tecnologia digital. Texto de la conferencia pronunciada en la $50^{a}$ Reunión del GEL -Grupo de Estudios Lingüísticos del Estado de São Paulo, USP, São Paulo, 23-25 de mayo de 2002.

Martín, M. y Portolés, J. (1999). Los marcadores del discurso. En I. Bosque y V. Demonte, Gramática descriptiva de la lengua española (Vol. 3, pp. 4051-4213). Madrid: Espasa Calpe.

Morales, M. (2007). Similitudes y diferencias entre texto e hipertexto. Aportes desde la lingüistica textual. Tesis de maestría. Universidad de Antioquia. Recuperado el 6 de marzo de 2010 de: http://www.tallerdecontenidos.com/wp-content/ uploads/2008/04/mauricio-moralessaldarriaga.pdf

Pavez, L. (2003). Damitas hermosas ¿por qué se esconden? Cortesía lingüistica e imagen en las salas de chat. Tesis de maestría en Lingüística. Universidad de Costa Rica.

Pérez, D. (2014). Mecanismos de cohesión textual en las conversaciones de Facebook. Tesis de maestría en Lingüística. Universidad de Costa Rica.

Pons, S. (1998). Conexión y conectores: estudio de su relación en el registro informal de la lengua. Valencia: Universidad de Valencia.

Ruiz, C. (4 de octubre de 2013). Cuarta parte de los usuarios de Facebook en Costa Rica ha cliqueado sus anuncios. La Nación (www.nacion. com).

Tascón, M. (ed.). (2012). Escribir en Internet: Guía para los nuevos medios y las redes sociales. Barcelona: Galaxia Gutenberg.

Tavares, I. (2008). A influência dos hiperlinks na leitura de hipertexto enciclopédico digital. Tesis de maestría. Universidad Federal de Minas Gerais. Recuperado el 28 de febrero de 2010 de: www.dominiopublico. gv.br

Van Dijk, T. (1983). La ciencia del texto. Barcelona: Editorial Paidós.

Wellman, B. (2001). Lugar físico y lugar virtual: El surgimiento de las redes personalizadas. En: Porras, J. y Espinoza V. (2005). Redes: enfoques y aplicaciones del análisis de redes sociales. Santiago, Chile: Ed. Universidad Bolivariana. 
